\title{
Going digital in dental sleep medicine
}

Obstructive Sleep Apnoea is a highly debilitating condition whereby the walls of the upper airway relax during sleep and obstruct breathing, leading to interrupted sleep. Treatment involves the fabrication of a special dental appliance that prevents the airway closing, something which is considerably easier when using a digital intraoral scanner with high precision such as the CS 3600 from Carestream Dental.

\section{The impact of sleep apnoea}

Paul Reaney, a dentist from Dentistry @ Markethill, explains the dangers

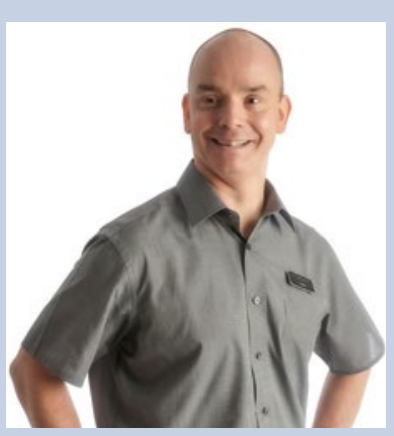

of sleep disorders and why the

CS 3600 intraoral scanner from

Carestream Dental is the ideal option for treating these patients:

'What many people don't realise is that the impact of conditions such as sleep apnoea is huge. Acute sleep loss can quickly lead to problems such as anxiety and stress - it takes the fun away from people's lives, damages relationships and this,

in turn, affects their productivity. Long-term effects can even be life threatening, as diabetes and heart attacks are just some of the conditions associated with lack of sleep that professionals need to be aware of.

'Unlike cosmetic dentistry where patients experience satisfaction based on aesthetics alone, effective sleep dentistry can literally change patients' lives, not only improving the quality but also the quantity of sleep.

'This is why I'm always looking to make a big difference for my patients, fast. By adopting a digital workflow and using an intraoral scanner instead of traditional impression techniques I can ensure that my patients get the care they need in a much shorter timescale and provide better outcomes.'

\section{Portable and precise}

'For me, one of the biggest benefits of the CS 3600 is the selection of tips', says Paul. 'It has the smallest tip available on the market, and as I work with a lot of younger patients for orthodontic care, this really helps me to treat everybody properly.

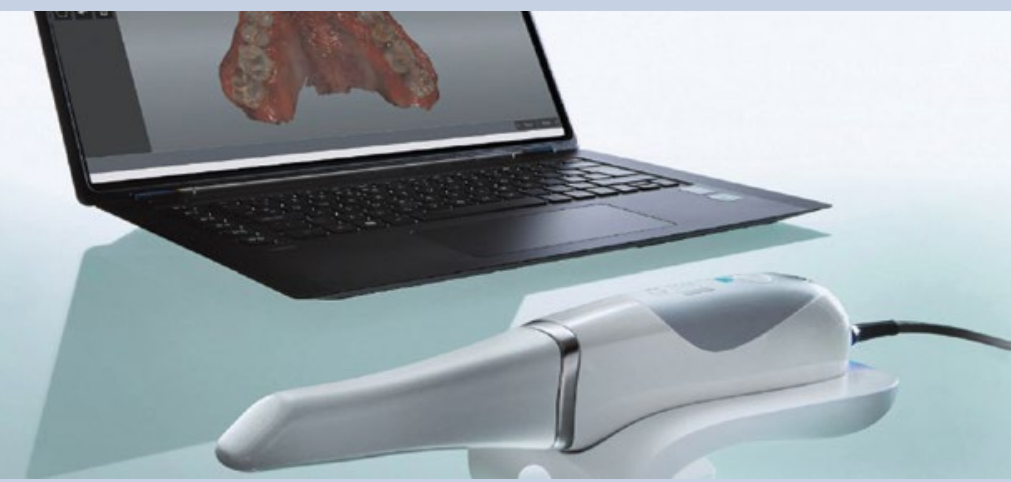

'The live scanning warnings are also incredibly helpful - they point out any areas of the scan that may need to be redone or filled in, and as I can send the scan to my technician as soon as it has been taken, I can easily retake it if necessary without my patient having to book another appointment. As I travel between clinics, the portable nature of the scanner is another huge benefit.'

\section{Achieving an accurate fit}

The appliances used to treat sleep apnoea have to be custom made to the individual, protruding the mandible so that the airway no longer becomes obstructed. Paul comments:

'The completely digital workflow helps me achieve patient satisfaction and streamline treatment at the same time. Patients love that they have access to a state-of-the-art treatment option, and this helps to build patient-practitioner trust.'

'Due to the CS 3600's accuracy, I can instantly assess a number of important details that are necessary for the fabrication of an effective appliance. Not only are the scans highly accurate, but I can also pick up details that are simply impossible to capture using traditional methods. Furthermore, as the CS 3600, in particular, has such a good level of trueness and I can use the scans to assess the depths of undercuts - a necessary measurement to ensure that appliances fit perfectly.'

'The Multiple Bite option is integral to providing Mandibular Advancement Appliances. This function allows me to take up to three different bite registrations that can be viewed in the software: for example, normal, open and protruded bites, without inaccuracies of recording the bite registration by traditional methods.'

\section{Ongoing excellence}

'One of the most important things to provide patients fitted with these appliances is continued support. Appliances can cause teeth to move over time, and with the CS 3600 I can take scans at review appointments and superimpose them to identify any tooth movement. This is also useful for patients with bruxism before receiving their appliance, as it is an effective way to gauge tooth wear when rescanned at reviews.'

\section{Always by your side}

'For me, one of the main benefits of the CS 3600 is that it's so easy to use. The software is intuitive and the wand itself is straightforward, helping the whole process to become stress-free. The support from Carestream Dental is also fantastic - they really provide that personal connection that you don't often get in the digital age, and that makes all the difference.'

The CS 3600 is suited to a wide array of indications and can help enhance outcomes as well as turnaround times and patient comfort.

For more information, contact Carestream Dental on 08001699692 or visit www.carestreamdental.co.uk. For the latest news and updates, follow us onTwitter@CarestreamDental and Facebook. 